\title{
Particle-In-Cell/Monte Carlo Simulation of Ion Back BomBardment in a High Average Current RF Photo-Gun
}

\author{
J. Qiang \\ Lawrence Berkeley National Laboratory, \\ Berkeley, CA 94720
}

\begin{abstract}
In this paper, we report on study of ion back bombardment in a high average current radio-frequency ( $\mathrm{RF}$ ) photo-gun using a particle-in-cell/Monte Carlo simulation method. Using this method, we systematically studied effects of gas pressure, RF frequency, RF initial phase, electric field profile, magnetic field, laser repetition rate, different ion species on ion particle line density distribution, kinetic energy spectrum, and ion power line density distribution back bombardment onto the photocathode. Those simulation results suggested that effects of ion back bombardment could increase linearly with the background gas pressure and laser repetition rate. The RF frequency has significantly affected the ion motion inside the gun so that the ion power deposition on the photocathode in an RF gun can be several orders of magnitude lower than that in a DC gun. The ion back bombardment can be minimized by appropriately choosing the electric field profile and the initial phase.
\end{abstract}

\section{INTRODUCTION}

A number of high average current RF photo-guns have been proposed or under development for future energy recovery linac (ERL) or free electron laser (FEL) applications [1-5]. At Lawrence Berkeley National Laboratory (LBNL), a normal conducting, very high frequency (VHF) RF gun operating at $187 \mathrm{MHz}$ is under construction [5]. This gun is designed to provide a high brightness electron beam up to $1 \mathrm{nC}$ charge with $1 \mathrm{MHz}$ repetition rate (i.e. $1 \mathrm{~mA}$ average current) to feed into a superconducting linac for a soft X-ray FEL array planned at LBNL [6]. At present, experiments at high average current photo-gun based on fixed voltage (i.e. DC gun) suggested that the ion back bombardment could cause significant decrease of quantum efficiency of the cathode in photoinjectors by either surface pollution or cathode material destruction [7-9]. It is a major factor that limits the photocathode lifetime in high average current photoinjectors.

In previous studies, single particle tracking and analytical model were used to estimate the ion generation and deposition on the cathode of the gun [10-12]. Those studies provided useful understanding of ion generation and dynamics inside the gun. However, for more accurate prediction of the ion back bombardment to the photocathode (e.g. ion particle distribution and energy spectrum on the cathode) under realistic conditions of the gun with electron beam, detailed numerical simulations are needed. In this paper, we used a self-consistent particle-in-cell/Monte Carlo method to study ion generation and ion back bombardment in the VHF RF photo-gun that is under construction at the LBNL.

The organization of the paper is as follows: after the introduction, we briefly describe the theoretical model of ion motion in an RF field in Section 2, introduce the computational model in Section 3, present simulations of the ion back bombardment of the VHF gun in Section 4, and summarize the study in Section 5. 


\section{THEORETICAL MODEL OF ION MOTION IN RF FIELD}

Inside an RF photo gun, when an ion is generated through electron impact ionization of residual gas, it will be subject to the acceleration of the electromagnetic fields of the cavity. For the slow motion of ion, the effects of magnetic fields are negligible. The equations of motion of an ion can be written as:

$$
m \ddot{\mathbf{r}}=q \mathbf{E}(\mathbf{r}) \cos (\omega \mathbf{t}+\phi)
$$

where $m$ is the mass of the ion, $q$ is the charge of the ion, $\mathbf{E}$ is the spatial dependence of electric field inside an RF cavity, $\omega$ is the angular frequency of the cavity, $\phi$ is the initial driven phase of the cavity. The solution of above equations of motion can be written as a summation of two terms $[12-16]$ :

$$
\mathbf{r}=\overline{\mathbf{r}}+\xi
$$

where $\overline{\mathbf{r}}$ denotes the slow drift term, and $\xi$ denotes the fast oscillating term. The solution of the fast oscillating term is given by

$$
\begin{aligned}
& \xi=-\frac{q \mathbf{E}(\mathbf{r}) \cos (\omega \mathbf{t}+\phi)}{m \omega^{2}} \\
& \dot{\xi}=\frac{q \mathbf{E}(\mathbf{r}) \sin (\omega \mathbf{t}+\phi)}{m \omega}
\end{aligned}
$$

The equations of motion for the slow drift term becomes

$$
\begin{aligned}
m \ddot{\overline{\mathbf{r}}} & =-\nabla U_{e f f}(r) \\
U_{e f f}(r) & =\frac{q^{2} \mathbf{E}^{2}(\mathbf{r})}{4 m \omega^{2}}
\end{aligned}
$$

subject to the initial conditions

$$
\begin{aligned}
\overline{\mathbf{r}}(0) & \doteq \mathbf{r}(\mathbf{0}) \\
\dot{\overline{\mathbf{r}}}(0) & =\dot{\mathbf{r}}(0)-\dot{\xi}(0) \\
\dot{\xi}(0) & =\frac{q \mathbf{E}(\mathbf{r}) \sin (\phi)}{m \omega}
\end{aligned}
$$

From the Eq. 5, we can see that the ion will drift towards the minimum of the effective potential $U_{\text {eff }}$, i.e. electric field intensity, independent of the sign of the charge. This suggests that if the electric field is peaked at the cathode, the ions generated inside the RF gun will drift away from the photocathode towards the minimum of the electric field. This is very different from the situation of the DC gun, where all the ions generated inside the gun will be pull back towards the photocathode. Since the total solution of the ion motion includes both the drift term and the fast oscillating term, only those ions originating near the cathode can reach the cathode from the fast oscillating motion or from a negative initial velocity pointing towards the cathode. A larger oscillation amplitude will in general result in more ions ending on the cathode. From Eq. 3, we can see that the oscillation amplitude goes down quickly with the RF frequency. This suggests that a smaller number of ions would come back to the cathode with a higher RF frequency. From Eqs. 8 and 9, we can see that the kinetic energy of the ion from the fast oscillation will also decrease with higher RF frequency, larger atomic mass and smaller absolute value of initial phase. 


\section{COMPUTATIONAL MODEL}

We have used the IMPACT-T code as the computational tool in this study. The IMPACT$\mathrm{T}$ code is a self-consistent three-dimensional quasi-static particle-in-cell (PIC) code to simulate charged particle transport in DC or RF photoinjectors [17]. Here, a bunch of photo-electrons emitted from the cathode will be transported through the injector subject to both the external accelerating/focusing forces and self-consistent space-charge forces. The electron beam collides with the background residual gas and produces ions through the electron-impact ionization. The production of ions is simulated using an Monte Carlo method with an ionization probability of the residual gas. Here, the probability of production of an ion by an electron impact ionization at each time step is given by [18]:

$$
p_{i}=1-\exp \left(-n_{\text {gas }} \sigma v d t\right)
$$

where $n_{\text {gas }}$ is the density of background residual gas, $\sigma$ is the electron impact ionization cross section, $v$ is the relative speed between the electron and the gas molecule, and $d t$ is the time step size. Given the ionization probability for an electron, a uniformly distributed random number $r$ is generated. If $r<p_{i}$, the ionization occurs, an ion particle is generated. Once an ion is generated, it will be subject to both the external forces of accelerating/focusing fields and the space-charge forces from the electrons. The space-charge forces among the ions and the space-charge forces on the electrons from the ions are neglected given the fact that the number of ions is much less than the number of electrons. The initial spatial location of the ion is assumed to be the same as that of the electron. The initial velocity of the ion is sampled from a Gaussian distribution with a given initial gas temperature. Here, we also neglect the detailed momentum exchange between the electron and the ion given the fact that this is small compared with the large external accelerating forces.

\section{SIMULATION OF ION BACK BOMBARDMENT IN THE VHF RF GUN}

Using the above particle-in-cell/Monte Carlo method, we have studied the ion back bombardment in the VHF RF photo-gun that is being developed at Lawrence Berkeley National Laboratory [5]. This VHF photo-gun is designed to provide a high average current electron beam with a good beam quality to support the soft X-ray FEL array planned at Berkeley [6]. The charge of electron beam from a single laser pulse can be as high as $1 \mathrm{nC}$ with a repetition rate of $1 \mathrm{MHz}$. The normal conducting RF gun cavity is designed with a nominal $187 \mathrm{MHz}$ frequency to combine the advantage of DC gun for high repetition rate and that of RF gun for higher accelerating field. The maximum field inside the gun is about $20 \mathrm{MV} / \mathrm{m}$ that provides the final electron beam energy around $750 \mathrm{keV}$. In the current study of the ion back bombardment inside this RF photo-gun, we have assumed a group of nominal parameters listed in the following table:

Table I: Nominal parameters of the $1 / 2$ cell VHF gun.

\begin{tabular}{lr}
\hline parameter & value \\
\hline Beam energy $(\mathrm{keV})$ & 750 \\
Peak field $(\mathrm{MV} / \mathrm{m})$ & 20 \\
RF frequncy $(\mathrm{MHz})$ & 187 \\
Charge/bunch $(\mathrm{nC})$ & 0.8 \\
Repitition rate $(\mathrm{MHz})$ & 1 \\
Initial pulse length $(\mathrm{ps})$ & 100 \\
Initial pulse radius $(\mathrm{mm})$ & 1 \\
Initial emittance $(\mathrm{mm}-\mathrm{mrad})$ & 0.25 \\
\hline
\end{tabular}


We have used the energy dependent electron impact ionization cross section for residual $\mathrm{H}_{2}$ gas, $\mathrm{H}_{2} \mathrm{O}$ gas and $\mathrm{CH}_{4}$ gas that are commonly observed in most RF photo-guns [10,19]. Figure 1 shows the electron impact ionization cross section for those three species gases [20-22]. It is seen that the ionization cross section reaches the maximum value around $50 \mathrm{eV}$ of electron kinetic energy and falls off quickly after $100 \mathrm{eV}$ for all three species of gas. Among these three species, the $\mathrm{CH}_{4}$ gas has the largest electron ionization cross section followed by the $\mathrm{H}_{2} \mathrm{O}$ gas. The $\mathrm{H}_{2}$ has the smallest ionization cross section, which is more than a factor of two less than the other two gas species.

\section{A. Effects of gas pressure}

In this study, we have assumed a background residual gas pressure of $10^{-6}$ Torr for most simulations. This is significantly higher than the vacuum gas pressure in the planned RF gun that could be as low as $10^{-11}$ Torr. Using such an artificially higher pressure is to increase the numerical statistics in the simulation since the production rate of ions is very low in the real RF gun and the number of ions will be too small during the period of simulation time of about 50 pulses (i.e. $50 \mu \mathrm{s}$ ). To check the effects of background gas pressure on the ion back bombardment, we ran simulations using the nominal gas pressure of $10^{-6}$ Torr, gas pressure of $0.5 \times 10^{-6}$ Torr, and of $2 \times 10^{-6}$ Torr. Figure 2 shows the pulse averaged $\mathrm{H}_{2}^{+}$ion line density and the ion energy distribution on the photocathode for the three gas pressures. Figure 3 shows the pulse averaged $\mathrm{H}_{2}^{+}$ion power line density distribution on the photocathode for the three gas pressures. Here the ion line particle density and power density of the half pressure case are multiplied by a factor of two while those of the doubled pressure case are divided by a factor of two. It is seen that after those scalings, all three cases show very close ion line density and power distributions. The ion kinetic energy distribution on the photocathode is also very close except somewhat larger fluctuation for the half pressure case due to the poorer numerical statistics. These results suggest that we could scale our simulation results linearly down to $10^{-11}$ Torr without changing the details of ion distribution on the photocathode.

\section{B. Effects of RF frequency}

From previous theoretical discussions, we see that the frequency of RF field can have significant impact on the ion particle dynamics inside the RF field. Figure 4 shows the pulse averaged total $\mathrm{H}_{2}^{+}$ion particle number back bombardment onto the cathode as a function of the RF frequency. Here, we have assumed the same electric field profile for all different frequencies in order to focus on the effect of the RF frequency. This will not be true in a real cavity in general but can still be a valid approximation for some low frequency cavities. From the Figure 4, it is seen that the number of ions back bombardment onto the cathode is significantly reduced inside the RF gun compared with that inside the 0 frequency DC gun case. With the increase of RF frequency, the of number of ions also decreases, which qualitatively agrees with the theoretical prediction. Figure 5 shows the pulse averaged $\mathrm{H}_{2}^{+}$ion particle line density distribution on the photocathode for different $\mathrm{RF}$ frequencies inside the gun. For the DC gun case, it shows a high peak particle line density at the center, which is consistent with the experimental observation [9]. It also has a wider spread of the distribution on the photocathode since all ions generated inside the gun will come back to the cathode eventually. Those ions generated at larger radial amplitude contribute to larger radial distribution on the cathode. The ion particle line densities in the RF guns are much less than that in the DC gun with a factor of 4 to 7 lower peak value. The density distribution is also much narrower on the cathode. This is because only the ions that are produced near the photocathode can reach the cathode. Most ions produced inside the RF gun will drift downstream towards the 


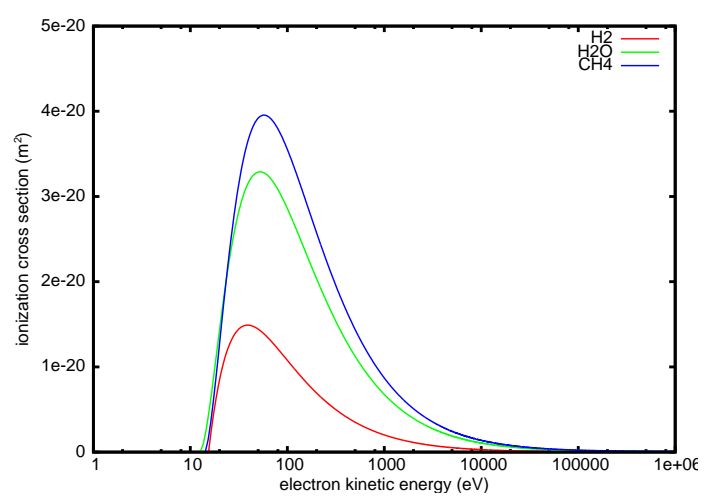

FIG. 1: Electron impact ionization cross section of $\mathrm{H}_{2}$ (red), $\mathrm{H}_{2} \mathrm{O}$ (green), and $\mathrm{CH}_{4}$ (blue) as a function electron kinetic energy.
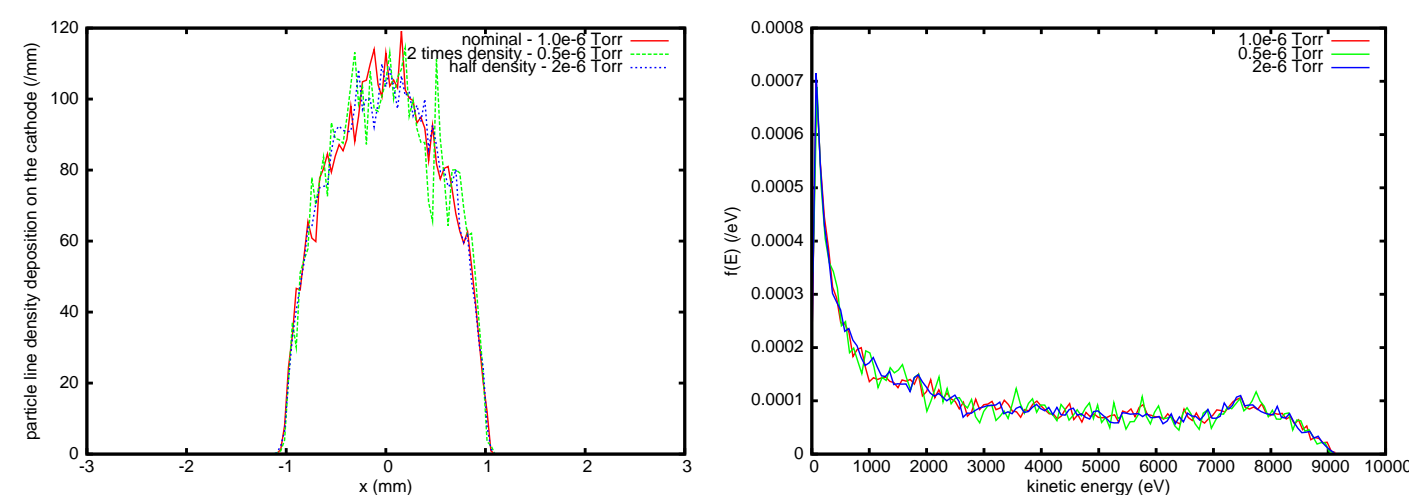

FIG. 2: Pulse averaged $H_{2}^{+}$ion particle line density distribution (top) and the kinetic energy distribution (bottom) on the photocathode with nominal pressure (red), half of the nominal pressure (green), and twice of the nominal pressure (blue).

exit of the gun. With the increase of the RF frequency, the amplitude of the fast ion oscillation decreases. This also results in less number of ions back onto the photocathode.

Figure 6 shows the $\mathrm{H}_{2}^{+}$ion kinetic energy distribution on the photocathode for different $\mathrm{RF}$ frequencies inside the gun. It is seen that inside the DC gun, the energy spectrum extends to a very wide range that corresponds to the maximum voltage across the accelerating gap. Also, the energy spectrum inside the DC gun has two peaks, one around the low energy regime, the other around the high energy regime. This is due to the fact that the ion production rate depends on the product of ionization cross section and the electron impact speed. The first peak near the low energy regime comes primarily from the contribution of large ionization cross section. The second peak around the high energy comes from the contribution of large electron impact speed down stream of the gun. Those high energy ions with more than $700 \mathrm{keV}$ kinetic energy could cause significant damage to the photocathode material. For the RF gun, the ion energy spread is much smaller than that of DC gun since only a small fraction of ions near the cathode can reach back the cathode. Most ions come back to the cathode with a low energy. For the $187 \mathrm{MHz}$ case, the maximum energy of ion is around $9000 \mathrm{eV}$ with most ions on the cathode have an energy below $100 \mathrm{eV}$. The impact damage of ions to the photocathode in the RF gun could be much less than that in the DC gun.

Figure 7 shows the $\mathrm{H}_{2}^{+}$ion power line density distribution on the photocathode for different $\mathrm{RF}$ frequencies inside the gun. It is seen that the power deposition inside the RF gun is several 


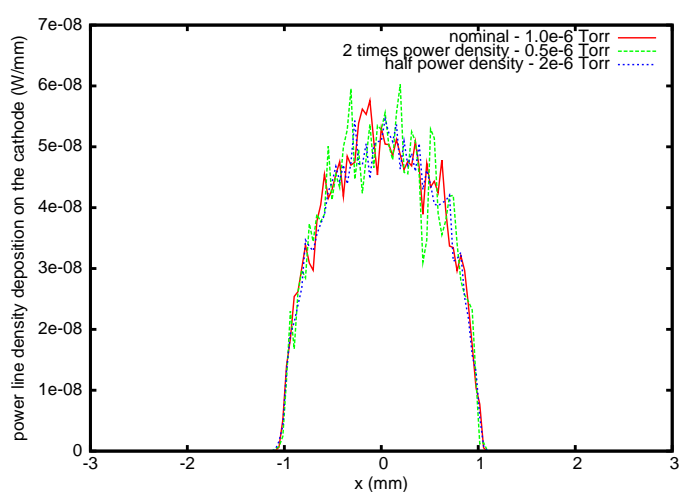

FIG. 3: Pulse averaged $H_{2}^{+}$power line density distribution on the photocathode with nominal pressure, half of the nominal pressure, and twice of the nominal pressure.

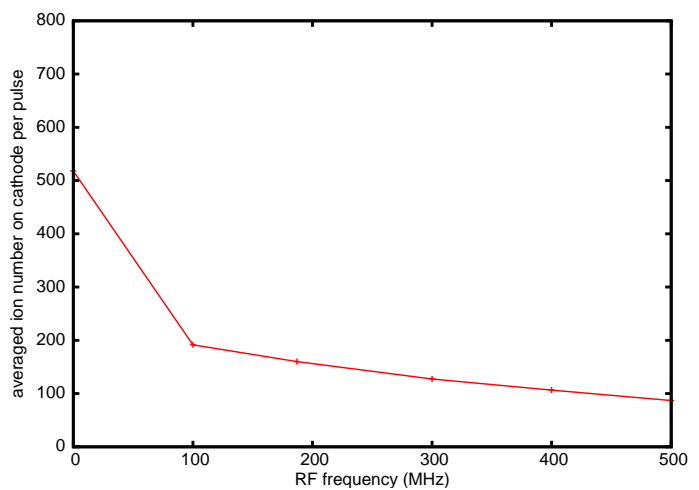

FIG. 4: The pulse averaged total $H_{2}^{+}$ion particle number on the cathode as a function of RF frequency.

orders of magnitude less than that in the DC gun.

\section{Effects of RF phases}

From the previous theoretical model it is also known that the velocity of ion motion in an RF field also depends on the initial phase of the RF field. Figure 8 shows pulse averaged $\mathrm{H}_{2}^{+}$ion particle line density distribution and the kinetic energy distribution on the photocathode with three different $\mathrm{RF}$ initial phases. Here the RF phase is defined with respect to the time of laser hitting onto the photocathode surface. It is seen that by adjusting the initial RF phase, the ion particle density on the photocathode can be lowered by $50 \%$ and the maximum ion kinetic energy impact on the cathode can also be reduced by $10 \%$. Figure 9 shows pulse averaged $\mathrm{H}_{2}^{+}$ion power line density distribution on the photocathode. The power line density can also be lowered by more than $50 \%$ by using a larger phase (i.e. smaller absolute value of phase).

Adjusting the initial RF phase could also affect the electron beam dynamics inside the RF gun. Figure 10 shows the electron beam kinetic energy evolution and transverse rms emittance evolution inside the gun with above three different initial RF phases. It is seen that the final energy and emittance are not very sensitive to the change of initial phase within this range of initial phase variation in this gun. This suggests that we could minimize the effects of ion back bombardment using a right phase without sacrificing the quality of electron beam output from the gun. 


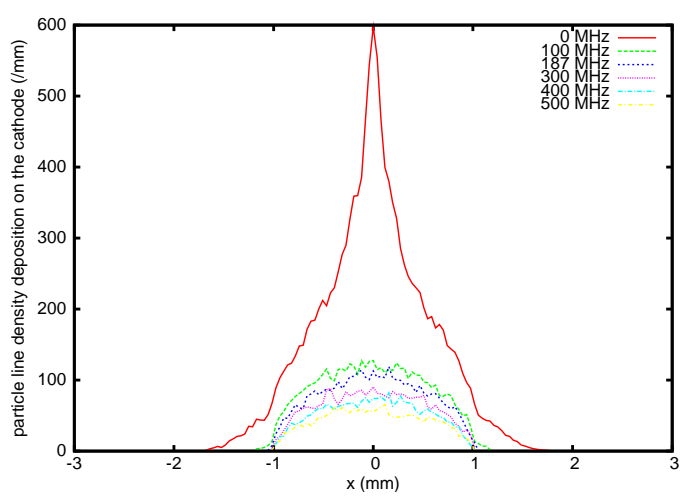

FIG. 5: Pulse averaged $H_{2}^{+}$ion particle line density distribution on the photocathode with 0 (red), 100 $\mathrm{MHz}$ (green), $187 \mathrm{MHz}$ (blue), $300 \mathrm{MHz}$ (pink), $400 \mathrm{MHz}$ (light blue), and $500 \mathrm{MHz}$ (yellow) RF frequencies inside the gun.
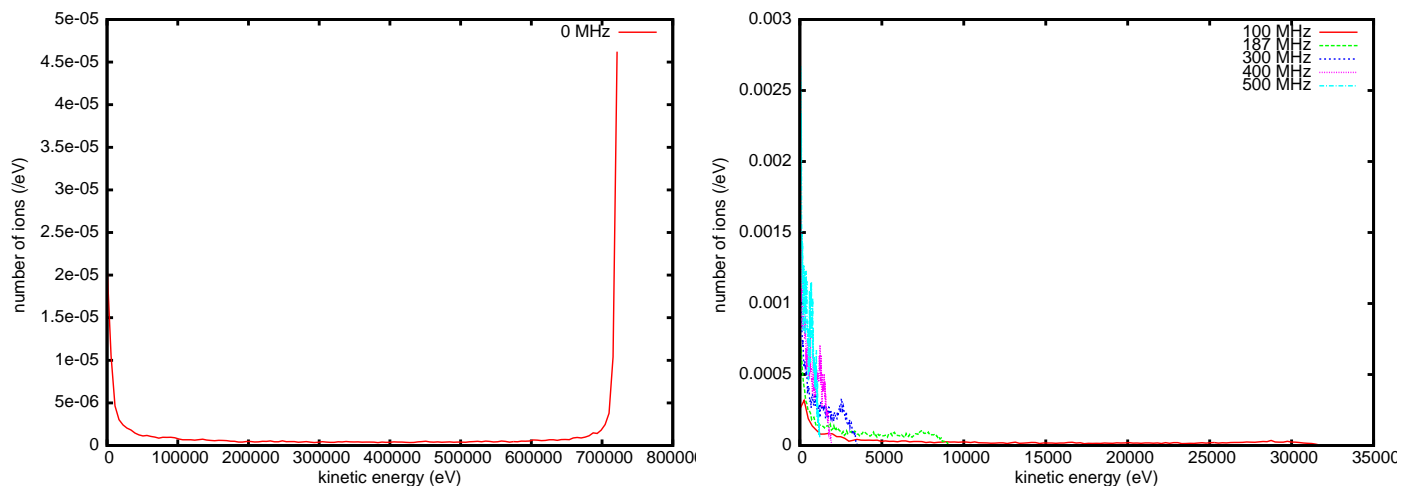

FIG. 6: Pulse averaged $H_{2}^{+}$kinetic energy distribution on the photocathode with 0 frequency (top), and with $100 \mathrm{MHz}$ (red), $187 \mathrm{MHz}$ (green), $300 \mathrm{MHz}$ (blue), $400 \mathrm{MHz}$ (pink), and $500 \mathrm{MHz}$ (light blue) RF frequencies inside the gun.

\section{Effects of RF field profile}

In photo-gun design, the cathode can be moved backward somewhat from the peak of the electric field in order to increase the transverse focusing of the electron beam from the electric field. However, from the previous theoretical model it is seen that ions inside the fast oscillation RF field will drift towards the minimum of electric field intensity. Moving the peak of field away from the cathode will cause some ions generated before the field peak location to drift towards the cathode. This would enhance the ion back bombardment to the photocathode. Figure 11 shows the pulse averaged $\mathrm{H}_{2}^{+}$ion particle line density distribution and the kinetic energy distribution on the photocathode with peak field at the cathode and at $1 \mathrm{~cm}$ away from the cathode. It is seen that the ion particle line density has increased significantly at the cathode. The ion kinetic energy distribution at the cathode also changes with more ions located near high energy regime. Figure 12 shows the pulse averaged $\mathrm{H}_{2}^{+}$ion power density distribution on the photocathode with peak field at the cathode and at $1 \mathrm{~cm}$ away from the cathode. It is seen that the ion power line density has increased by about a factor of two using the field with recessed cathode. 


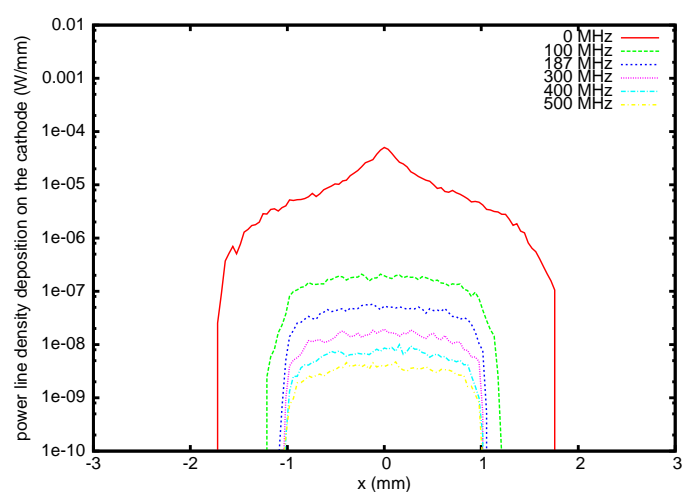

FIG. 7: Pulse averaged $H_{2}^{+}$ion power line density distribution on the photocathode with 0 (red), $100 \mathrm{MHz}$ (green), $187 \mathrm{MHz}$ (blue), $300 \mathrm{MHz}$ (pink), $400 \mathrm{MHz}$ (light blue), and $500 \mathrm{MHz}$ (yellow) RF frequencies inside the gun.
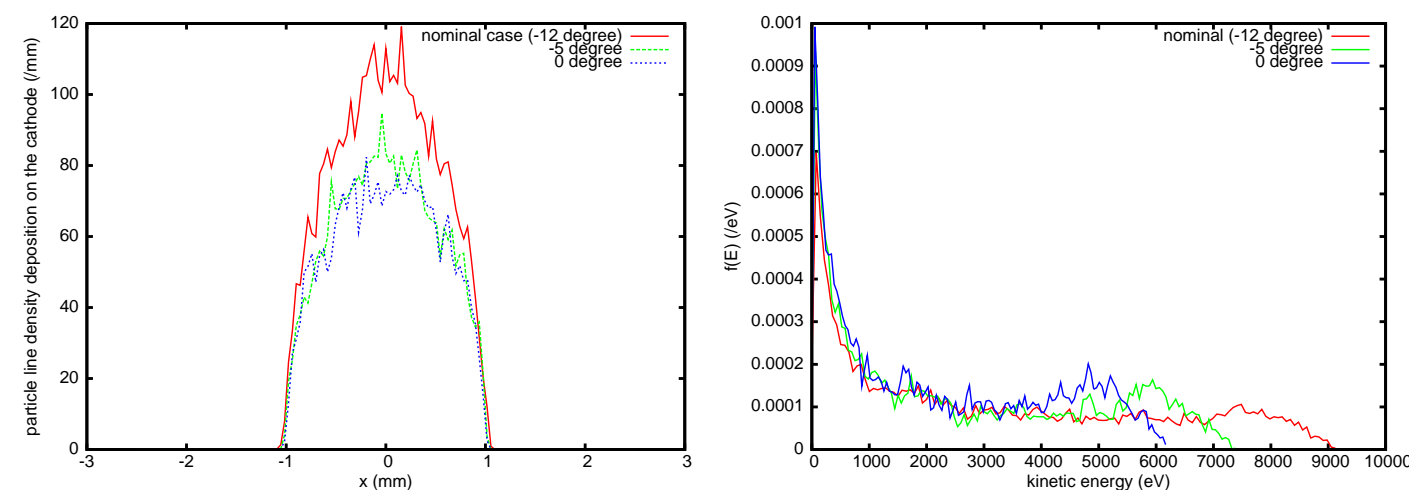

FIG. 8: Pulse averaged $H_{2}^{+}$ion particle line density distribution (top) and the kinetic energy distribution (bottom) on the photocathode with 0 degree (blue), -5 degree (green), and -12 degree (red) initial RF phases.

\section{E. Effects of repetition rate}

The VHF RF photo gun designed at LBNL can in principle also operate at even higher repetition rate than the nominal case of $1 \mathrm{MHz}$. Figure 13 shows the pulse averaged $\mathrm{H}_{2}^{+}$ion particle line density distribution and the kinetic energy distribution on the photocathode with $1 \mathrm{MHz}, 10 \mathrm{MHz}$, and $100 \mathrm{MHz}$ repetition rate. Figure 14 shows the pulse averaged ion power line density distribution on the cathode with above three repetition rates. It is seen that the ion particle line density and energy distribution are very close for all three repetition rates. The ion power line density increases linearly with the increase of the repetition rate. This suggests that running the photo RF gun with higher repetition rate would result in more ions back bombardment on the photocathode within the same amount of time than the nominal repetition rate. The ion distribution on the photocathode remains nearly the same.

\section{F. Effects of B field}

The solenoid magnetic field is used for the emittance compensation in order to control the emittance growth of the electron beam. Figure 13 shows the pulse averaged $\mathrm{H}_{2}^{+}$ion particle line density distribution and the kinetic energy spectrum on the photocathode without and with magnetic field inside the gun. Figure 14 shows the pulse averaged $H_{2}^{+}$ion power line density distribution on the 


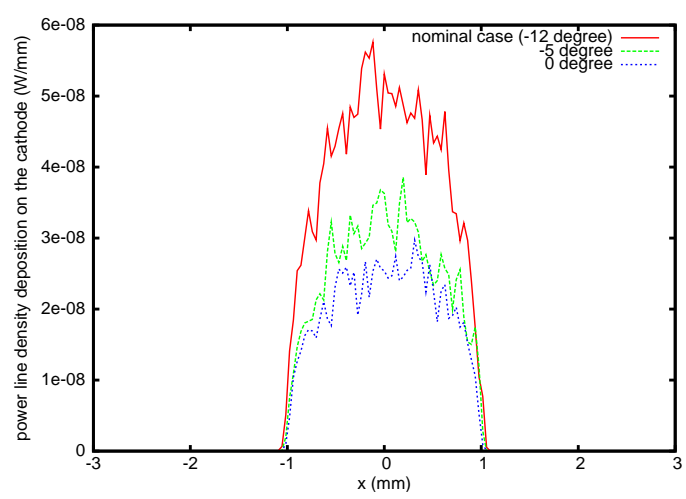

FIG. 9: Pulse averaged $H_{2}^{+}$ion power line density distribution on the photocathode with 0 degree (blue), -5 degree (green), and -12 degree (red) initial RF phases.
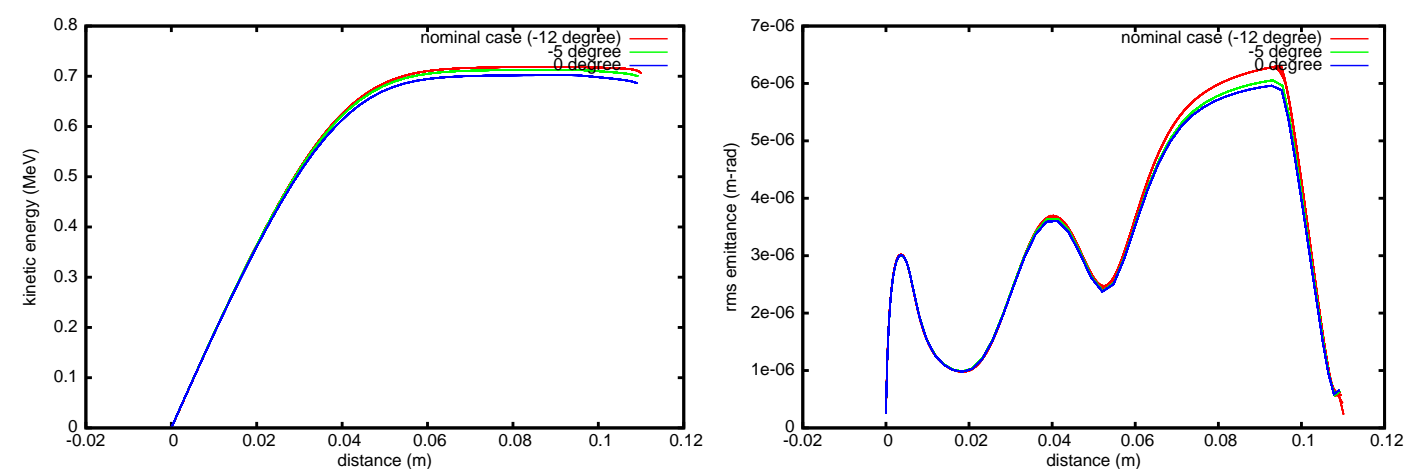

FIG. 10: Electron beam kinetic energy evolution (top) and transverse rms emittance evolution (bottom) inside the gun with 0 (blue), -5 (green), -12 (red) degree initial RF phases.

photocathode without and with magnetic field inside the gun. It is seen that there are no significant differences in the particle density distribution, power line density distribution, and kinetic energy spectrum on the cathode with or without magnetic field. The effects of magnetic field inside the gun on the ion back bombardment are small. This is due to both small ion velocity near the cathode and the small magnetic field near the cathode.

\section{G. Effects of ion species}

In the RF gun, the residual gas contains not only $H_{2}$ but also other species such as water and $\mathrm{CH}_{4}$. Those species can have different charge to mass ratio compared with the $\mathrm{H}_{2}^{+}$. Figure 17 shows the pulse averaged ion particle line density distribution and the kinetic energy distribution on the photocathode for $\mathrm{H}_{2}^{+}, \mathrm{CH}_{4}^{+}$and $\mathrm{H}_{2} \mathrm{O}^{+}$. It is seen that $\mathrm{CH}_{4}^{+}$has the largest particle line density on the cathode among three species due to the largest ionization cross section shown in Fig. 1. However, the $H_{2}^{+}$shows a very wide energy spectrum with a maximum kinetic energy up to $9000 \mathrm{eV}$. This is because the $\mathrm{H}_{2}^{+}$is the lightest ion among the three species and has a higher kinetic energy inside the gun. Figure 18 shows the pulse averaged ion power line density distribution on the photocathode for $\mathrm{H}_{2}^{+}, \mathrm{CH}_{4}^{+}$and $\mathrm{H}_{2} \mathrm{O}^{+}$. The $\mathrm{H}_{2}^{+}$has the largest power deposition on the photocathode due to its larger kinetic energy compared with the other two species. These results suggest that the back ion energy distribution on the photocathode is primarily determined by the ion mass while the ion particle density distribution depends heavily on the electron impact 

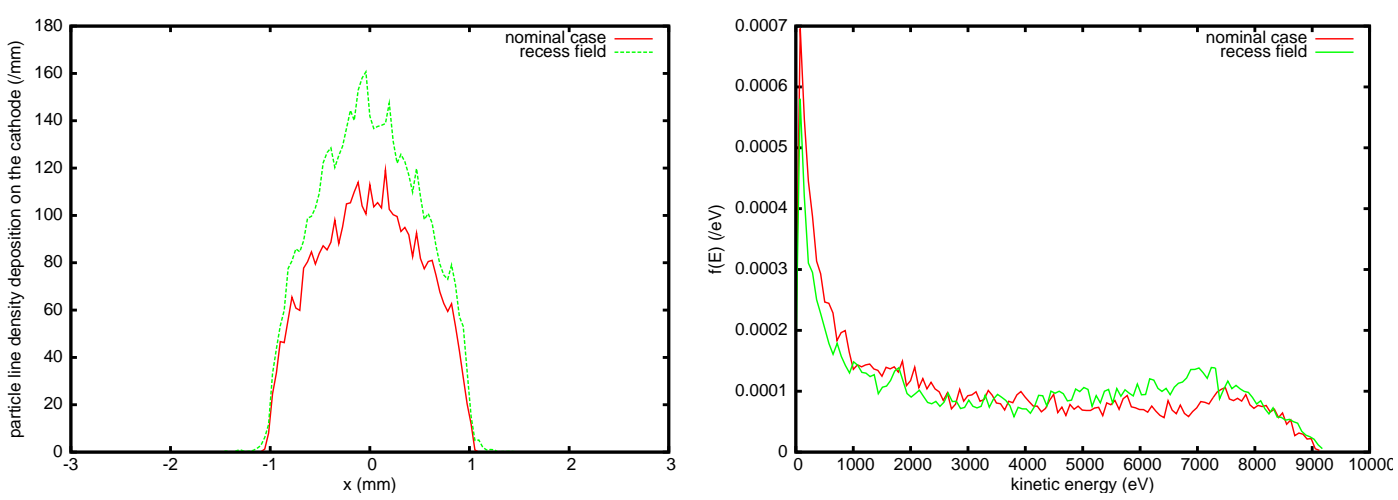

FIG. 11: Pulse averaged $H_{2}^{+}$ion particle line density distribution (top) and the kinetic energy distribution (bottom) on the photocathode with peak electric field on the cathode (red) and recessed electric field on the cathode (green).

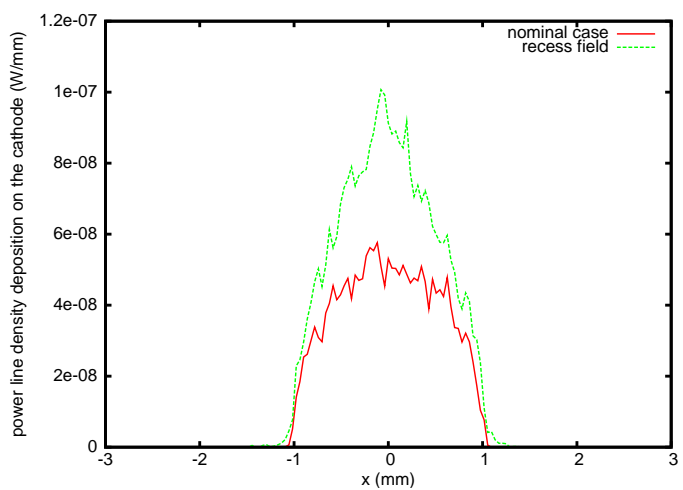

FIG. 12: Pulse averaged $H_{2}^{+}$ion power line density distribution on the photocathode with peak electric field on the cathode (red) and recessed electric field on the cathode (green).

ionization cross section.

\section{SUMMARY}

The above simulations studied the effects of residual gas pressure, RF gun frequency, RF field profile, RF initial phase, magnetic field, gas species, and laser repetition rate on the ion back bombardment to the photocathode in the VHF gun. These results suggest that ion back bombardment in the high average current RF photo gun could be much less than that in the DC gun. If the damage to the photocathode is primarily due to the surface contamination by the deposition of ion particles, the lifetime of the photocathode in the RF gun could be a few times better than that in the DC gun. If the damage to the photocathode is primarily due to the high energy ion back collision, the lifetime of the photocathode in the RF gun could be order of magnitude better than that in the DC gun. Since the ion particle density scales linearly with residual gas pressure, for the VHF photo gun operating with a vacuum pressure as low as $10^{-11}$ Torr, the real number of ions on the cathode could be $10^{-5}$ smaller than the nominal simulation case with a residual gas pressure of $10^{-6}$ Torr. If the ion particle would accumulate linearly with time, there could be about thousand ions on the cathode per week in the VHF RF gun. Increasing the repetition rate, i.e. increasing the average current, will lead to more ion back bombardment on the photocathode without significantly changing the particle and energy distribution. The simulation results also 

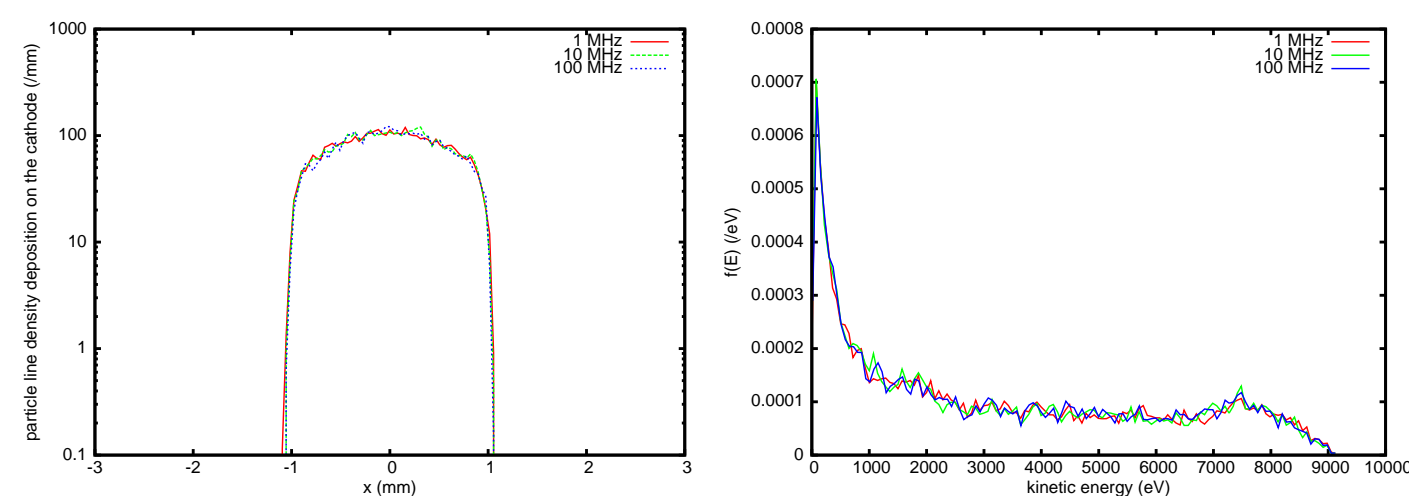

FIG. 13: Pulse averaged $H_{2}^{+}$ion particle line density distribution (top) and the kinetic energy distribution (bottom) on the photocathode with $1 \mathrm{MHz}$ (red), $10 \mathrm{MHz}$ (green), and $100 \mathrm{MHz}$ (blue) repetition rate.

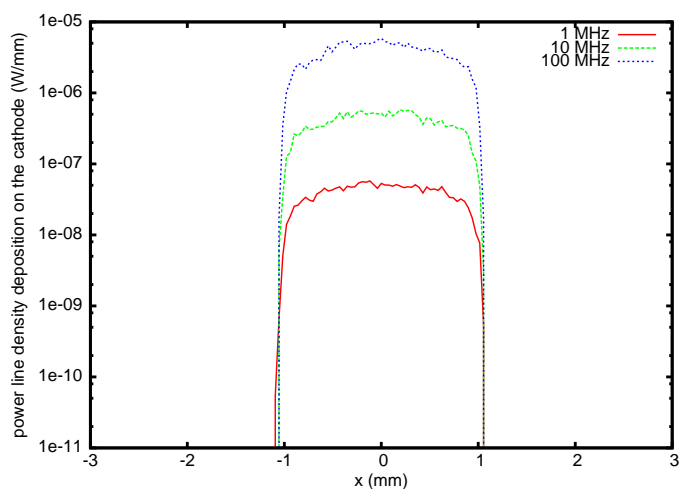

FIG. 14: Pulse averaged $H_{2}^{+}$ion power line density distribution on the photocathode with $1 \mathrm{MHz}$ (red), 10 $\mathrm{MHz}$, and $100 \mathrm{MHz}$ repetition rate.

suggested that the ion back bombardment could be minimized by appropriately choosing the RF electric field profile and the RF initial phase.

\section{ACKNOWLEDGEMENTS}

We would like to thank J. Corlett, S. Lidia and J. Staple, F. Sannibale for bringing the author into this topic, providing cavity field profile, and helpful discussions. This research was supported by the Office of Science of the U.S. Department of Energy under Contract No. DE-AC0205CH11231. This research used resources of the National Energy Research Scientific Computing Center.

\section{REFERENCES}

[1] J. Teichert et al., in Proceedings of EPAC 2004, Lucerne, Switzerland, p. 333 (2004).

[2] S. Kurennoy et al., in Proceedings of PAC 2005, Knoxville, Tennessee, USA, p. 2866 (2005).

[3] I. Ben-Zvi et al., in Proceedings of FEL 2007, Novosibirsk, Russia, p. 290 (2007).

[4] R. Legg, W. Graves, T. Grimm, P. Piot, in Proceedings of EPAC08, Genoa, Italy, p. 469 (2008). 

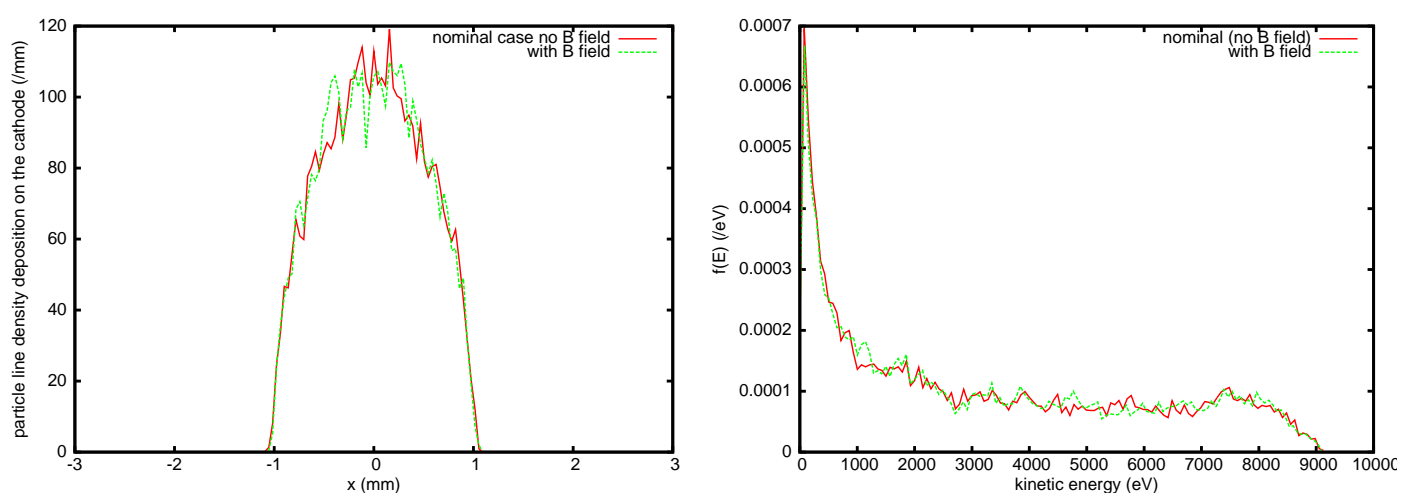

FIG. 15: Pulse averaged $H_{2}^{+}$ion particle line density distribution (top) and the kinetic energy distribution (bottom) on the photocathode without external magnetic field (red) and with magnetic field (green).

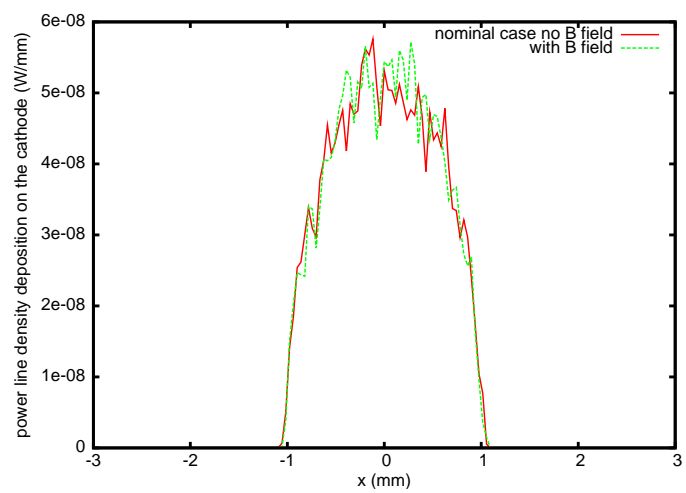

FIG. 16: Pulse averaged $H_{2}^{+}$ion power line density distribution on the photocathode without external magnetic field (red) and with magnetic field (green).

[5] K. Baptiste, J. Corlett, S. Kwiatkowski, S. Lidia, J. Qiang, F. Sannibale, K. Sonnad, J. Staples, S. Virostek, R. Wells, Nucl. Instrum. Methods Phys. Res., A 599, 9 (2009).

[6] J. N. Corlett et al., in Proceedings of PAC07, Albuquerque, New Mexico, USA, p. 1167 (2007).

[7] K. Aulenbacher et al., Nucl. Instrum. Methods Phys. Res., A 319, 498 (1997).

[8] J. Grames et al., in Proceedings of PAC05, Knoxville, Tennessee, USA, p. 2875 (2005).

[9] C. K. Sinclair et al., Phys. Rev. ST Accel. Beams 10, 023501 (2007).

[10] J. W. Lewellen, Phys. Rev. ST Accel. Beams 5, 020101 (2002).

[11] E. Pozdeyev, Phys. Rev. ST Accel. Beams 10, 083501 (2007).

[12] E. Pozdeyev, D. Kayran, V. N. Litvienko, Phys. Rev. ST Accel. Beams 12, 043501 (2009).

[13] P. L. Kapitza, Zh. Eksp. Teor. Fiz. 21, 588 (1951).

[14] L. D. Landau and E. M. Lifshitz, Mechanics (Course of Theoretical Physics) (ButterworthHeinemann, Burlington, MA, 1982), 3rd ed.

[15] A.V. Gaponov and M. A. Miller, Zh. Eksp. Teor. Fiz. 34, 242 (1958) (Sov. Phys. JETP 7, 168 (1958)).

[16] A. Ridinger and N. Davidson, Phys. Rev. A 76, 013421 (2007).

[17] J. Qiang, S. Lidia, R. D. Ryne, and C. Limborg-Deprey, Phys. Rev. ST Accel. Beams 9, 044204 (2006).

[18] J. Wang, P. Liewer, and E. Huang, J. Supercomputing, 10, 331 (1997).

[19] H. F. Dylla, "Challenges for extreme high Vacuum (XHV)," CERN Accelerator School, Platja D’Aro, Spain, May 16-24, 2006. 

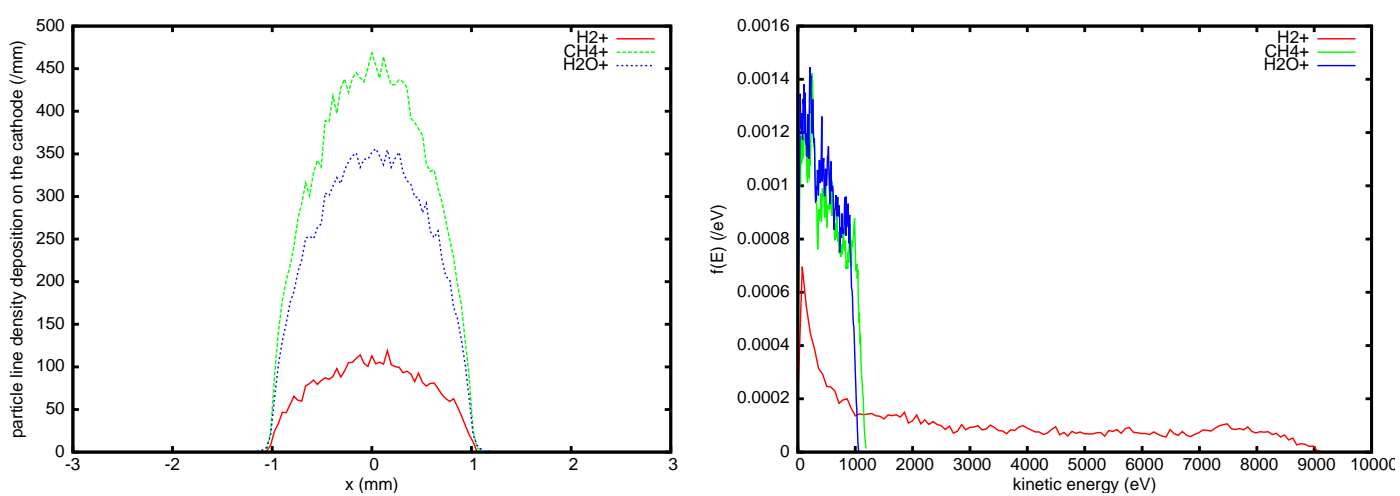

FIG. 17: Pulse averaged $H_{2}^{+}$ion particle line density distribution (top) and the kinetic energy distribution (bottom) on the photocathode for $\mathrm{H}_{2}^{+}$(red), $\mathrm{CH}_{4}^{+}$(green) and $\mathrm{H}_{2} \mathrm{O}^{+}$(blue) ions.

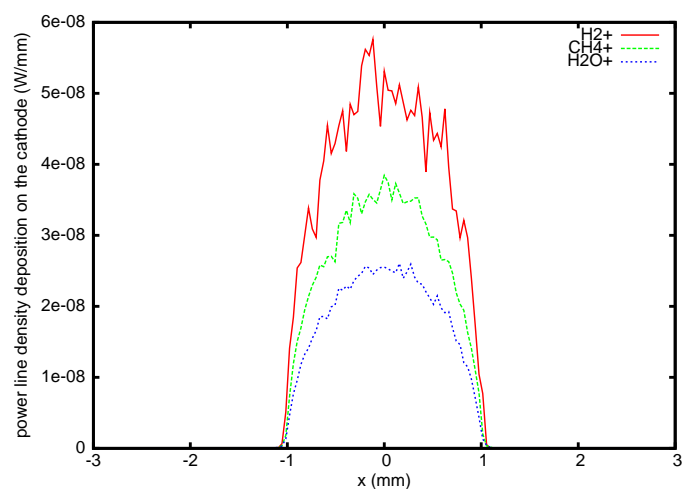

FIG. 18: Pulse averaged $\mathrm{H}_{2}^{+}$ion power line density distribution on the photocathode for $\mathrm{H}_{2}^{+}$(red), $\mathrm{CH}_{4}^{+}$ (green) and $\mathrm{H}_{2} \mathrm{O}^{+}$(blue) ions.

[20] F. F. Rieke and W. Prepejchal, Phys. Rev. A 6, 1507 (1972).

[21] S. P. Slinker, R. D. Taylor, and A. W. Ali, J. Appl. Phys. 63, 1 (1988).

[22] M. Reiser, Theory and Design of Charged Paricle Beams, Joh Wiley \& Sons, Inc., New York, 1994. 